\title{
Die WSI-Betriebsrätebefragung 2007 - Methoden und ausgewählte Ergebnisse
}

Die im Spätherbst 2007 erneut durchgeführte WSI-Hauptbefragung von Betriebsräten hat wieder repräsentative Daten gebracht zu den betrieblichen Problemen und Lösungswegen der Interessenvertretungen, zu den Rahmenbedingungen ihrer Arbeit und ihrem Verhältnis zu Arbeitgeber, Belegschaft und Gewerkschaft. Eines der zentralen Ergebnisse lautet, dass die betrieblichen Probleme, mit denen die Betriebsräte konfrontiert sind, trotz konjunkturellem Aufschwung seit 2005 nicht geringer geworden sind; teilweise - insbesondere bei den Arbeitsbedingungen - sich sogar verschärft haben. Alle Ergebnisse betreffen zwölf Millionen Arbeitnehmer und Arbeitnehmerinnen, die in Betrieben ab 20 Beschäftigten von einem Betriebsrat vertreten werden.

\section{Anlage und Methode}

Zum fünften Mal insgesamt und zum zweiten Mal mit der Methode des computergestützten Telefoninterviews (CATI) hat das WSI im Jahr 2007 eine Welle von Befragungen bei Betriebs- und Personalräten durchgeführt. Sie wurde in mehrere Teile gegliedert: Zunächst wurde die Befragung von Personalräten separiert und als Sonderbefragung ins Frühjahr 2007 vorgezogen. Deren Ergebnisse werden gesondert publiziert. Die Hauptbefragung im Spätherbst 2007 bezog sich allein auf die Betriebsräte. Doch auch hier mussten über die Basisbefragung hinaus einzelne Fragenmodule aus inhaltlichen wie organisatorischen Gründen ausgegliedert werden: So gab es einen schriftlich versandten Fragebogen zur betrieblichen Beschäftigungsstruktur (drop off-Fragebogen), der das Telefoninterview von Zahlenangaben entlasten sollte. Zudem wurde eine thematisch eingeschränkte Zusatzbefragung durchgeführt, um geringe Fallzahlen bei bestimmten Themen der Basisbefragung durch weitere Interviews aufzustocken.

Die Sorge um geringe Fallzahlen war jedenfalls ein Grund für eine Betriebsrätebefragung mit sehr großer Brutto-Stichprobe. Zu diesem Zweck zogen wir aus der Betriebsstättendatei der Bundesagentur für Arbeit (BA) eine Stichprobe von rund 67.000 Betrieben ab 20 sozialversicherungspflichtigen Beschäftigten (ca. ein Drittel der Grundgesamtheit). Die Ziehung der Stichprobe war disproportional geschichtet, um in vorgegebenen Wirtschaftsbereichen und Betriebsgrößenklassen eine Mindestzahl von Interviews pro Ziehungszelle zu erhal-

\begin{tabular}{|c|c|c|c|c|c|}
\hline ... in Wirtschaftsbereichen & Betriebe & $\begin{array}{l}\text { Beschäf- } \\
\text { tigte }\end{array}$ & $\begin{array}{l}\text {... in } \\
\text { Betriebsgrößen- } \\
\text { klassen }{ }^{2} \text { ) }\end{array}$ & Betriebe & $\begin{array}{l}\text { Beschäf- } \\
\text { tigte }\end{array}$ \\
\hline Grundstoffe/Produktionsgüter & 43,9 & 76,0 & \multirow{2}{*}{5 bis 19} & \multirow{2}{*}{ k. A. } & \multirow{2}{*}{ k. A. } \\
\hline Investitions-/Gebrauchsgüter & 38,8 & 77,4 & & & \\
\hline Verbrauchsgüter & 35,7 & 64,3 & 20 bis 49 & 18,3 & 19,6 \\
\hline Baugewerbe & 18,5 & 35,4 & 50 bis 99 & 38,2 & 39,3 \\
\hline Handel/Reparatur & 28,8 & 46,4 & 100 bis 199 & 58,1 & 58,7 \\
\hline Verkehr/Nachrichten & 26,1 & 48,9 & 200 bis 499 & 74,8 & 75,6 \\
\hline $\begin{array}{l}\text { Private Kreditinstitute/ } \\
\text { Versicherungen }{ }^{1} \text { ) }\end{array}$ & 66,2 & 77,4 & 500 bis 999 & 84,4 & 84,8 \\
\hline Weitere private Dienste ${ }^{1)}$ & 25,6 & 42,2 & 1000 bis 1999 & 88,1 & 88,7 \\
\hline sonstige Branchen ${ }^{3)}$ & 65,8 & 80,4 & 2000 und mehr & 85,9 & 89,5 \\
\hline Gesamt $^{4)}$ & 31,6 & 58,9 & Gesamt $^{4)}$ & 31,6 & 58,9 \\
\hline \multicolumn{6}{|c|}{$\begin{array}{l}\text { 1) In \% der erreichten Betriebsstätten aus der Stichprobe, anders als die vergleichbare Tabelle in Schäfer (2005) ohne Personalräte } \\
\text { - 2) Nach Anzahl der Beschäftigten. - 3) Vor allem Bergbau, chemische Produkte, Energieerzeugung. - 4) } 7.688 \text { von } 32.210 \text { be- } \\
\text { fragten Betrieben. }\end{array}$} \\
\hline
\end{tabular}

ten. Nach Zuspielung von Telefonnummern und Abzug der neutralen Ausfälle wurden 32.210 Betriebe gefragt, ob sie einen Betriebsrat haben. Das bejahten 7.688 Betriebe. Unter diesen wurden in zwei getrennten Unterstichproben 2.070 vollständige Telefoninterviews für die Basisbefragung und 1.816 Kurzinterviews für die $\mathrm{Zu}-$ satzbefragung (zu Namenslisten bei betriebsbedingten Entlassungen) realisiert. Trotz der Dauer (im Durchschnitt 50 Minuten für Basisinterviews und 15 Minuten für Zusatzinterviews) erfuhr der Befragungsprozess wieder eine hohe Akzeptanz bei den Befragten (zumeist Betriebsratsvorsitzende): Nur 31 Interviews wurden endgültig abgebrochen; gut $90 \%$ aller Interviewten haben sich zu weiteren Interviews bereit erklärt.

Die große Bruttostichprobe von 32.210 kontaktierten Betrieben ermöglicht uns, noch zuverlässiger als bisher die Häufigkeit von Betriebsratsgremien in Betrieben ab 20 Beschäftigten zu erfassen (Tabelle 1). Dies gilt im Vergleich zur letzten Welle (Schäfer 2005), aber auch in Relation zur Arbeitgeberbefragung des Instituts für Arbeitsmarkt- und Berufsforschung (IAB), das mit einer kleineren Erhebung (rund 15.000 Betriebe) seine Daten auf ein größeres Segment der Betriebslandschaft, nämlich alle betriebsratsfähigen Betriebe ab fünf Beschäftigte, hochrechnen muss (vgl. Ellguth/ Kohaut 2007).

Die von der WSI-Befragung erfassten 7.688 Betriebe stehen für fast ein Drittel aller Betriebe ab 20 Beschäftigte in Deutschland und einen viel höheren Anteil an Arbeitsplätzen, weil die mittleren und vor allem die großen Betriebe eine überpro-

Claus Schäfer, Dr., ist Wissenschaftler im WSI in der Hans-Böckler-Stiftung und Koordinator der WSI-Arbeitsgruppe Befragungen. Arbeitsschwerpunkt: Verteilungsanalyse von Einkommen und Lebenslagen. e-mail: claus-schaefer@boeckler.de 
portionale Beschäftigtenzahl auf sich konzentrieren. Absolut stehen die von der WSIBefragung abgebildeten Ergebnisse für rund zwölf Millionen Beschäftigte.

\section{Eckdaten der Befragung}

Im Folgenden werden einige wichtige Ergebnisse aus der Betriebsrätebefragung 2007 präsentiert. Sie sind teils ungewichtet, wenn die Datenstruktur vorgestellt wird und teils gewichtet, wenn inhaltlich repräsentative Ergebnisse referiert werden. Von Interesse ist zunächst die Verteilung der geführten Interviews auf Regionen, Wirtschaftsbereiche, Betriebsgrößen und gewerkschaftliche Organisationsbereiche. Der Vergleich mit der Vergangenheit wird wegen der damaligen Umstellung auf CATI auf die 4. Befragungswelle von 2005 beschränkt.

Tabelle 2 zeigt die Verteilung der befragten Betriebsräte und ihrer Betriebe auf Regionen, die zwischen den Befragungen kaum Unterschiede aufweist. Die Fallverteilung von Basis- und Zusatzbefragung unterscheidet sich bei anderen Merkmalen nur unwesentlich stärker, sodass hier auf die Zusatzbefragung nicht weiter eingegangen wird. Die Verteilung auf Beschäftigungsgrößenklassen gibt Tabelle 3 wieder. Hier zeigt der Zeitvergleich, dass die 2007 realisierte größere Bruttostichprobe ermöglicht hat, die 2005 schwach besetzten unteren Betriebsgrößen entsprechend ihrer Häufigkeit viel stärker, d.h. realitätsgerechter, abzubilden.

Laut Tabelle 4 hat auch die Verteilung der realisierten Interviewfälle auf Wirtschaftsbereiche eine Verschiebung der Häufigkeiten im Vergleich zu 2005 ergeben, die der realen Verteilung von Betriebsstätten besser entspricht. Im Zuge dieser Verschiebung bei den Wirtschaftsbereichen ändert sich auch die Verteilung der durchgeführten Interviews auf die gewerkschaftlichen Organisationsbereiche (Tabelle 5). Im Rahmen der dabei erfassten Betriebe mit Betriebsräten gibt es auch „gewerkschaftsfreie" Zonen, die nach Angaben der befragten Betriebsräte von keiner Gewerkschaft organisiert werden. Allerdings ist ihr Anteil an allen Betrieben mit Betriebsrat recht gering $(5,3 \%)$ - und aufgrund der Fragestellung vermutlich noch geringer als ausgewiesen.
Tabelle 2: Fallzahlen1) der WSI-Befragungen nach Regionen

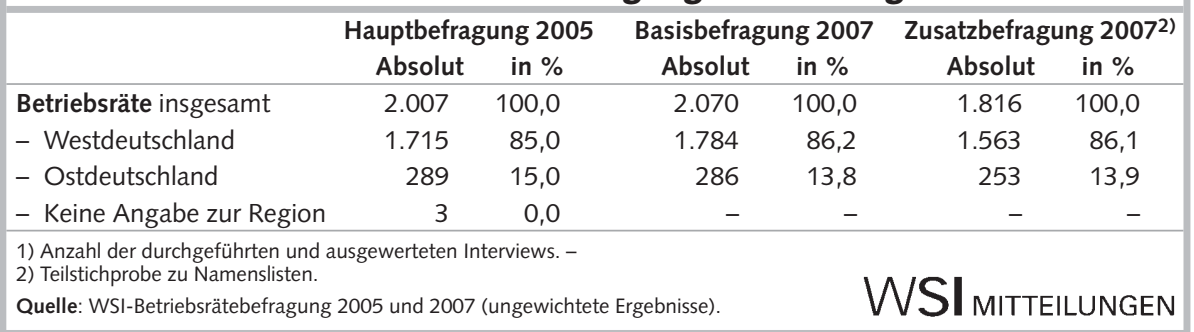

Tabelle 3: Befragte Betriebsräte nach Betriebsgrößenklassen

\begin{tabular}{|lcrrr|}
\hline Größenklasse & \multicolumn{2}{c}{ 2005 } & \multicolumn{2}{c|}{ 20071) } \\
& Absolut & in \% & Absolut & in \% \\
\hline bis 49 Beschäftigte & 72 & 3,6 & 1.056 & 51,0 \\
50 bis 99 Beschäftigte & 167 & 8,3 & 291 & 14,1 \\
100 bis 199 Beschäftigte & 367 & 18,3 & 224 & 10,8 \\
200 bis 499 Beschäftigte & 534 & 26,6 & 199 & 9,6 \\
über 500 Beschäftigte & 867 & 43,2 & 300 & 14,5 \\
\hline Insgesamt & $\mathbf{2 . 0 0 7}$ & $\mathbf{1 0 0 , 0}$ & $\mathbf{2 . 0 7 0}$ & $\mathbf{1 0 0 , 0}$ \\
\hline 1) Hauptbefragung 2007; nur Teilstichprobe Basisbefragung. & & W SI MITTEILUNGEN \\
Quelle: WSI-Betriebsrätebefragung 2005 und 2007 (ungewichtete Ergebnisse). & W SI MITEN
\end{tabular}

\begin{tabular}{|c|c|c|c|c|}
\hline \multirow[t]{2}{*}{ Wirtschaftsbereich } & \multicolumn{2}{|c|}{2005} & \multicolumn{2}{|c|}{ 20071) } \\
\hline & Absolut & in $\%$ & Absolut & in $\%$ \\
\hline Grundstoffverarbeitung & 337 & 16,8 & 160 & 7,7 \\
\hline Investitionsgüter & 441 & 22,0 & 293 & 14,2 \\
\hline Verbrauchsgüter & 246 & 12,3 & 222 & 10,7 \\
\hline Baugewerbe & 96 & 4,8 & 118 & 5,7 \\
\hline Handel (u. Reparatur) & 158 & 7,9 & 367 & 17,7 \\
\hline Verkehr, Nachrichtenübermittlung & 131 & 6,5 & 143 & 6,9 \\
\hline Private Kreditinstitute2)/Versicherungen & 181 & 9,0 & 119 & 5,7 \\
\hline weitere private Dienstleistungen²) & 240 & 12,0 & 556 & 26,9 \\
\hline sonstige Branchen & 176 & 8,8 & 92 & 4,4 \\
\hline Keine Angabe von Branchen & 1 & 0,0 & - & - \\
\hline Insgesamt & 2.007 & 100,0 & 2.070 & 100,0 \\
\hline \multicolumn{5}{|c|}{$\begin{array}{l}\text { 1) Hauptbefragung 2007; nur Teilstichprobe Basisbefragung. -2) } 2005 \text { waren auch öffentliche Banken und öffentliche } \\
\text { Dienstleistungen eingeschlossen. } \\
\text { Quelle: WSI-Betriebsrätebefragung } 2005 \text { und } 2007 \text { (ungewichtete Ergebnisse). }\end{array}$} \\
\hline
\end{tabular}

\begin{tabular}{|c|c|c|c|c|}
\hline \multirow[t]{2}{*}{ Organisationsbereich ${ }^{1)}$} & \multicolumn{2}{|c|}{2005} & \multicolumn{2}{|c|}{ 20072) } \\
\hline & absolut & in $\%$ & absolut & in $\%$ \\
\hline IG BAU & 118 & 5,9 & 132 & 6,4 \\
\hline ver.di & 792 & 39,5 & 918 & 44,3 \\
\hline IGM & 640 & 31,9 & 548 & 26,5 \\
\hline Transnet & 16 & 0,8 & 11 & 0,5 \\
\hline NGG & 93 & 4,6 & 85 & 4,1 \\
\hline IG BCE & 283 & 14,1 & 158 & 7,6 \\
\hline GEW & 4 & 0,2 & 13 & 0,6 \\
\hline Sonstige & 9 & 0,4 & 80 & 3,9 \\
\hline Keine Gewerkschaft & 27 & 1,3 & 110 & 5,3 \\
\hline weiß nicht & 25 & 1,2 & 14 & 0,7 \\
\hline verweigert & 0 & 0 & 1 & 0,0 \\
\hline Insgesamt & 2.007 & 100,0 & 2.070 & 100,0 \\
\hline \multicolumn{5}{|c|}{$\begin{array}{l}\text { 1) Interviewfrage: Von welcher Gewerkschaft wird Ihre Belegschaft überwiegend organisiert? - 2) Hauptbefragung 2007; } \\
\text { nur Teilstichprobe Basisbefragung. } \\
\text { Quelle: WSI-Betriebsrätebefragung } 2005 \text { und } 2007 \text { (ungewichtete Ergebnisse). }\end{array}$} \\
\hline
\end{tabular}


Tabelle 6: Betriebliche Problembereiche und Problembedeutung 2005-2007

\begin{tabular}{|c|c|c|c|}
\hline Betriebliche Probleme1) & $\begin{array}{l}\text { Problem } \\
\text { existent } \\
\text { (Häufigkeit in \%) }\end{array}$ & $\begin{array}{l}\text { Intensitäts- } \\
\text { rate2) } \\
\text { (Mittelwert) }\end{array}$ & $\begin{array}{l}\text { Prioritätsrate }{ }^{3)} \\
\text { (Häufigkeit in \%) }\end{array}$ \\
\hline Personalabbau / Beschäftigungssicherung & 60,8 & 2,3 & 14,9 \\
\hline Altersteilzeit & 58,8 & 2,9 & 3,7 \\
\hline Sozialplan / Interessenausgleich & 31,5 & 2,3 & 4,9 \\
\hline Einschränkung der Ausbildung & 19,9 & 2,9 & 0,2 \\
\hline $\begin{array}{l}\text { Ausgliederung / Schließung oder } \\
\text { Zusammenlegung von Betriebsteilen }\end{array}$ & 33,8 & 2,4 & 6,7 \\
\hline Änderung der Arbeitsorganisation & 58,0 & 2,7 & 3,7 \\
\hline Arbeitsbedingungen Älterer & 38,2 & 3,0 & 0,5 \\
\hline Familienfreundliche Arbeitsbedingungen & 40,5 & 3,0 & 1,0 \\
\hline $\begin{array}{l}\text { Gleichstellung von Männern und Frauen / } \\
\text { Frauenförderung }\end{array}$ & 26,7 & 3,9 & 0,1 \\
\hline Einführung neuer Techniken & 54,2 & 2,7 & 2,5 \\
\hline Arbeitsschutz / Gesundheitsförderung & 77,0 & 2,8 & 1,8 \\
\hline Fort- und Weiterbildung & 69,9 & 2,9 & 1,1 \\
\hline Erhöhung des Leistungsdrucks & 70,9 & 2,5 & 5,5 \\
\hline Arbeitszeitkonten & 67,0 & 2,5 & 10,3 \\
\hline Zielvereinbarungen / Mitarbeitergespräche & 66,9 & 2,7 & 4,1 \\
\hline mehr Überstunden & 64,8 & 2,4 & 9,4 \\
\hline mehr Wochenendarbeit & 38,0 & 2,7 & 2,9 \\
\hline betriebliche Altersversorgung / Riesterrente & 61,8 & 2,9 & 1,8 \\
\hline Einschränkung betrieblicher Sozialleistungen & 32,8 & 2,8 & 1,6 \\
\hline Abbau übertariflicher Leistungen & 36,9 & 2,6 & 3,4 \\
\hline $\begin{array}{l}\text { Unterschreitung von Tarifstandards / } \\
\text { Kürzung tariflicher Leistungen }\end{array}$ & 37,0 & 2,3 & 5,7 \\
\hline $\begin{array}{l}\text { Wünsche der Beschäftigten nach flexiblen } \\
\text { Arbeitszeiten }\end{array}$ & 47,2 & 2,7 & 2,7 \\
\hline Verschlechterung des Betriebsklimas & 64,3 & 2,6 & 4,9 \\
\hline $\begin{array}{l}\text { Mangelnder Rückhalt des Betriebsrats } \\
\text { bei den Beschäftigten }\end{array}$ & 33,0 & 3,0 & 1,9 \\
\hline Allgemeines Gleichbehandlungsgesetz von 2006 & 54,0 & 3,2 & 0,3 \\
\hline Neues Elternzeitgesetz von 2006 & 26,2 & 3,6 & 0,0 \\
\hline \multicolumn{4}{|c|}{$\begin{array}{l}\text { 1) Interviewfrage: Welche Entwicklungen und Probleme haben den BR seit Anfang } 2005 \text { besonders beschäftigt? (vorgegebene } \\
\text { Antwortmöglichkeiten, Mehrfachnennungen möglich). - 2) Interviewfrage: Wie intensiv war die Beschäftigung mit diesem } \\
\text { Thema auf einer Skala von } 1 \text { - sehr intensiv - bis } 6 \text { - überhaupt nicht intensiv? - 3) Interviewfrage: In welchem Bereich lag das } \\
\text { größte Problem für die Betriebsratsarbeit seit Anfang 2005? }\end{array}$} \\
\hline $\begin{array}{l}\text { Quelle: WSI-Betriebsrätebefragung } 2007 \text { (gewichtete Ergebnis } \\
\text { Basisbefragung). }\end{array}$ & se/nur Teilstichprobe & & MITTEILUNGEN \\
\hline
\end{tabular}

Wegen der insgesamt realitätsnäheren Verteilung der Fallzahlen sind auch die Interviewergebnisse zuverlässiger geworden. Insbesondere die Gewichtung der Ergebnisse relativiert sich: Die Ziehung der Adressstichprobe bei der Bundesagentur für Arbeit war schon - neben dem Kriterium einer Mindestzellenbesetzung - möglichst proportional zur realen Verteilung der Betriebe auf Betriebsgrößen und Wirtschaftsbereiche erfolgt, wie sie die Betriebsstättendatei der BA für alle Betriebe ausweist. Die Differenzen der Ziehungswahrscheinlichkeiten zwischen Verteilung der Grundgesamtheit und Verteilung der verwirklichten Interviews auf Betriebsgrößen und Wirtschaftsbereiche werden pro Stichprobenzelle und Ziehungsschritt als Gewichte für die Interviewergebnisse genutzt (Horvitz-Thompson-Schätzer). Da die Differenzen geringer geworden sind, fallen die Gewichte kleiner aus als früher.
Frage nach der Intensität der Beschäftigung des Betriebsrats mit einem Problem ( $\mathrm{Ta}$ belle 6).

Ein weiterer Indikator für die Intensität der Betriebsratsaktivität zu einem bestimmten Thema ist der Neuabschluss von Betriebsvereinbarungen, der auch in den früheren Befragungen erhoben wurde ( $\mathrm{Ta}$ belle 7). Aus den in beiden Tabellen präsentierten Indikatoren lassen sich Schwerpunkte der Aktivitäten ableiten, auch wenn die benannten Themen nicht deckungsgleich sind.

Schon die Quantität der betrieblichen Problemlagen wie auch die Fülle der Lösungs- oder Kanalisierungsversuche der Betriebsräte, die am prägnantesten in Betriebsvereinbarungen zum Ausdruck kommen, ist bemerkenswert. Bei den Betriebsvereinbarungen kommt hinzu, dass sie mit vorausgehendem Verhandlungsprozess und hoher rechtlicher Verbindlichkeit den meisten Arbeitsaufwand wie den größten Erfolg darstellen dürften. Fast sieben Betriebsvereinbarungen existieren heute im Durchschnitt pro Betrieb, davon wurde nahezu die Hälfte (3,1 pro Betrieb) seit Anfang 2005 abgeschlossen, d.h. aktualisiert oder inhaltlich erstmalig erarbeitet. Nicht überraschend ist eine mit der Betriebsgröße eindeutig wachsende Zahl von Vereinbarungen: in kleinen Betrieben mit 20 bis 49 Beschäftigten gibt es durchschnittlich 6,7 (neu: 2,9), in Betrieben ab $2.000 \mathrm{Be}-$ schäftigten 12,9 (neu: 5,5).

Gemessen am "größten Problem" ragt erneut der explizite Personalabbau bzw. die Beschäftigungssicherung als Aktivitätsspitze heraus (Prioritätsrate 14,9\%). Dabei taucht dieses Problem - ohne große Unterschiede im Zeitvergleich - implizit auch bei anderen benannten Problembereichen mit hoher Häufigkeit bzw. Bedeutung auf: bei Sozialplan und Interessenausgleich (Prioritätsrate 4,9\%) - was bei betroffenen Betrieben trotz Aufschwung auf besonders massiven Personalabbau hindeutet; bei Altersteilzeit als Form des Personalabbaus; bei Ausgliederung und Schließung oder Zusammenlegung von Betriebsteilen ( $\mathrm{Ta}$ belle 6). Der Neuabschluss von Betriebsvereinbarungen zur „Beschäftigungssicherung " bei immerhin rund $20 \%$ der befragten Betriebe unterstreicht die Wichtigkeit dieses Komplexes von teils faktischem, teils befürchtetem Personalabbau und den Versuch, ihn zu kanalisieren.

Einen vergleichbar bedeutenden Aktivitätskomplex bilden die Bereiche Ände- 
rung der Arbeitsorganisation (bei rund $60 \%$ der befragten Betriebe) und Erhöhung des Leistungsdrucks (bei rund $70 \%)$. Er erfährt eine konkrete Ausprägung in der Arbeitszeitregulierung (Tabelle 6): Arbeitszeitkonten - d. h. Einführung, Änderung oder Auseinandersetzung um solche Konten - weisen bei einer Häufigkeit von zwei Dritteln aller befragten Betriebe eine Prioritätsrate von 10,3 \% auf, die eindeutig zweithöchste nach dem Personalabbau. $\mathrm{Zu}$ dieser Einschätzung passt, dass mehr Überstunden und sogar mehr Wochenendarbeit nicht nur bei zwei Dritteln bzw. einem Drittel der Betriebe seit Anfang 2005 vorkommen, sondern teils auch mit einer sehr hohen Prioritätsrate bei den befragten Betriebsräten versehen werden (9,4 \% bei „Mehr Überstunden“, Tabelle 6). Auch die Neuabschlüsse von Betriebsvereinbarungen zu Arbeitszeitkonten und zu Mehrarbeit - auf der Basis eines schon hohen Bestands an solchen Vereinbarungen betonen die Bedeutung der Arbeitszeit für die Betriebsratsaktivitäten (Tabelle 7): $\mathrm{Zu}$ beiden Themen gab es die meisten Neuabschlüsse von Betriebsvereinbarungen (Rang 1 bzw. Rang 3) bei gut bzw. knapp einem Drittel aller befragten Betriebe.

Es scheint trotz dieser und weiterer teils schwieriger Problemlagen doch erstaunlich, dass fast zwei Drittel aller befragten Betriebsräte eine Verschlechterung des Betriebsklimas melden (64,3\%). Die dazu genannte Prioritätsrate liegt mit 4,9\% ebenfalls auf einem der oberen Ränge. Zur Erklärung können die weiteren Ergebnisse der Betriebsrätebefragung einen Teil beitragen.

\subsection{EINZELERGEBNISSE}

Die vertiefenden Interviewfragen sind in Einzelkomplexe gegliedert: Beschäftigung und Beschäftigtenstruktur im Betrieb, betriebliche Umstrukturierung/Standortverlagerung, Arbeitszeit, Lohn/Gehalt/Entgelt, Tarifbindung, Beziehungen zwischen Betriebsrat, Arbeitgeber, Belegschaft und Gewerkschaft sowie schließlich Struktur und Organisation des Betriebsrats selbst. Auf Grund der regelmäßigen Wiederholung entsprechender Detailfragen in den Befragungswellen sind Zeitvergleiche möglich, wenn auch teilweise eingeschränkt durch Änderungen der Befragungsmethode, Stichprobenstruktur und Fragestellung. Darüber hinaus kamen aktualitätsbezogene Fragen ergänzend oder als eigenständi-

\begin{tabular}{|c|c|c|}
\hline Inhalt der Vereinbarung & $\begin{array}{l}\text { Bestehende } \\
\text { Vereinbarung1) }\end{array}$ & $\begin{array}{c}\text { Davon neue } \\
\text { Vereinbarung }\end{array}$ \\
\hline Einstellungen & 25,0 & 10,3 \\
\hline Entlassungen & 14,8 & 7,8 \\
\hline Versetzung / Umstrukturierung & 22,9 & 11,4 \\
\hline Eingruppierung & 29,8 & 12,0 \\
\hline Entlohnung & 35,0 & 17,7 \\
\hline Zielvereinbarungen & 26,0 & 15,7 \\
\hline Arbeitszeitverkürzungen & 13,2 & 6,2 \\
\hline Arbeitszeitverlängerungen & 28,0 & 15,8 \\
\hline Arbeitszeitkonten & 58,4 & 26,3 \\
\hline Mehrarbeit & 46,6 & 20,6 \\
\hline Ausbildung & 22,3 & 9,5 \\
\hline Weiterbildung/Qualifizierung & 34,9 & 14,5 \\
\hline Familienfreundlichkeit & 9,7 & 3,6 \\
\hline Gleichstellung von Frauen und Männern/Frauenförderung & 11,9 & 5,3 \\
\hline Technikgestaltung & 21,1 & 11,1 \\
\hline Arbeitsorganisation & 25,7 & 11,5 \\
\hline Arbeitsschutz & 37,5 & 13,1 \\
\hline Beschäftigungssicherung & 26,5 & 15,1 \\
\hline Sucht & 26,7 & 11,0 \\
\hline Datenschutz & 52,7 & 23,0 \\
\hline Betriebliche Sozialleistungen & 44,1 & 14,2 \\
\hline Vorschlagswesen & 34,0 & 11,9 \\
\hline Arbeitsbedingungen älterer Beschäftigter & 10,2 & 3,3 \\
\hline Vorzeitiges Ausscheiden älterer Beschäftigter & 23,2 & 8,3 \\
\hline Ganzheitliche Gefährdungsbeurteilung & 19,7 & 9,9 \\
\hline Insgesamt: Anzahl Betriebsvereinbarungen pro Betrieb & 6,9 & 3,1 \\
\hline \multicolumn{3}{|c|}{$\begin{array}{l}\text { 1) Interviewfrage: Zu welchem der folgenden Regelungsbereiche gibt es (Gesamt-)Betriebsvereinbarungen? } \\
\text { (vorgegebene Antwortmöglichkeiten, Mehrfachnennungen möglich). } \\
\text { 2) Interviewfrage: Wurde diese Betriebsvereinbarung seit } 2005 \text { neu abgeschlossen? }\end{array}$} \\
\hline Quelle: WSI-Betriebsrätebefragung 2007 (gewichtete Ergebnisse/nur Basis! & & MITTEILUNC \\
\hline
\end{tabular}

ge Themenkomplexe ins Interview, diesmal zu dem betrieblichen Umgang mit jüngeren und älteren Beschäftigten, dem Einsatz von Leiharbeitnehmern, der Mitarbeiterkapitalbeteiligung, der Nutzung neuer Mitwirkungsrechte von Betriebsrat und Belegschaft und den Namenslisten bei betriebsbedingten Entlassungen. Einige Ergebnisse werden hier zusammenfassend vorgestellt:

\section{BESCHÄFTIGUNGS- UND BESCHÄFTIGTENPROBLEME}

Der betriebliche Einsatz von Leiharbeit (Seifert/Brehmer in diesem Heft) generiert besondere Belastungen der Betriebsratsarbeit. $23 \%$ der Interessenvertretungen berichten von Auseinandersetzungen mit dem jeweiligen Arbeitgeber wegen der Leiharbeit - und in fast jedem zehnten Betrieb (9\%) gab es Konflikte zwischen Betriebsrat und Verleihfirma. Ein überraschend hoher Anteil der Betriebsräte erklärt, dass sie mit Leiharbeitnehmern - formalrechtlich keine Betriebsbeschäftigten - im Vergleich zur regulären Belegschaft einen gleichen oder sogar größeren Arbeitsaufwand haben, insbesondere in den Feldern ,arbeitsvertragliche Regelungen“ (43\%), ,Arbeits- und Gesundheitsschutz“ (76 \%), „Regelung der Arbeitszeiten“ (69\%), „Besuch von Leiharbeitnehmern in den Sprechstunden des Betriebsrats“ (60 \%), und „Abschluss von Betriebsvereinbarungen für Leiharbeitnehmer" (46 \%) (ähnlich Wassermann/Rudolph 2007). Angesichts solcher Ergebnisse scheint die Entscheidung des Bundesarbeitsgerichts fragwürdig, dass Leiharbeitnehmer bei der Berechnung von Mandaten und Freistellungen des Betriebsrats nicht mitzählen sollen.

Weit weniger prominent ist die Lage von Berufseinsteigern in den ersten Jahren ihrer regulären Erwerbstätigkeit. Die Auskünfte der Betriebsräte zu dieser Beschäftigtengruppe legen dagegen angesichts der erkennbaren Probleme bei ihrem betrieblichen Einsatz weit mehr öffentliche Sensibilität nahe. Die Berufseinsteiger wurden in drei Gruppen nach personalplanerischen Voraussetzungen und Zielsetzungen ihres Einsatzes differenziert und bei den Betriebsräten abgefragt:

(1) Trainees sind häufig Akademiker, die im Betrieb auf der Grundlage ihrer Qualifikation eine befristete praxisorientierte, betriebsspezifische Einarbeitung bzw. Weiter- 
qualifizierung erfahren, bevor sie auf einem meist vor Trainingsbeginn in Aussicht gestellten Arbeitsplatz eingesetzt werden sollen. Eine hohe, betrieblich geschätzte, mitgebrachte Ausbildung wird mit einem spezifischen betrieblichen Weiterbildungsprogramm aufgestockt, um ein möglichst großes Humankapital für den Betrieb zu gewinnen.

(2) Mit Praktikanten dagegen ist normalerweise die weit weniger ambitionierte $\mathrm{Ab}$ sicht verknüpft, Betriebspraxis nach einer vorangegangenen betriebsexternen Qualifizierung zu vermitteln, ohne die Zielsetzung einer späteren Übernahme in eine normale Beschäftigung.

(3) Sonstige Berufseinsteiger ohne Traineeprogramm oder Praktikum sind zwischen den beiden erstgenannten Einsteigerkategorien angesiedelt; sie werden für eine bestimmte Tätigkeit im Betrieb eingestellt und müssen sich dafür zunächst bewähren und eine gewisse Einarbeitung akzeptieren.

Aktuell setzen $21 \%$ der Betriebe Trainees, $28 \%$ Praktikanten und sogar $38 \%$ Berufseinsteiger im oben genannten Sinn ein. Dabei nehmen die Betriebe häufig die Heranführung an die betriebliche Praxis nicht ernst genug und verknüpfen nicht selten vollwertigen Arbeitseinsatz mit unterwertigen Arbeitsbedingungen.

Bei Trainees bestätigt sich zwar der erwartete hohe Grad an betrieblicher Verbindlichkeit: Drei Viertel der Betriebe mit Trainee-Einsatz bieten einen festen und personenbezogenen betrieblichen Betreuer für die Trainee-Zeit an, genauso viele Betriebe haben einen fixierten Betreuungsplan. Demzufolge bewerten fast alle dazu befragten Betriebsräte ( $93 \%$ ) ihre jeweiligen Trainee-Stellen als ein „Lernverhältnis“ bzw. ein „Heranführen an die betriebliche Praxis“. Allerdings räumt gut die Hälfte von ihnen (54\%) ein, dass es sich beim TraineeVerhältnis eher um eine normale Beschäftigung handele. Ein Fünftel der Betriebsräte $(21 \%)$ melden für Trainees eine Bezahlung unterhalb des Tarifs für Normalbeschäftigte. In beiden Mitteilungen kommt ein betriebsseitiges Flexibilitätsinteresse, hier bei den Kosten, an anderer Stelle bei der Einsatzdauer zum Vorschein. So berichten die Betriebsräte, dass auch bei Trainees - trotz der generellen Befristung fast immer eine Probezeit zwischen drei und sechs Monaten vereinbart wird. Der
Abbruch des Trainee-Verhältnisses während der Probezeit wurde nicht erfragt. Aber fast jeder fünfte Betrieb (19\%) übernahm seit Anfang 2005 keinen einzigen der seitdem eingesetzten Trainees nach Abschluss ihrer Qualifizierung; die restlichen Betriebe stellten einen beachtlichen Anteil der jeweiligen Trainees nach Trainingsabschluss ein. Aber selbst von den übernommenen Trainees erhielt die Hälfte nur einen befristeten Anstellungsvertrag.

Zum Einsatz von Praktikanten fallen viele abgefragte Indikatoren erwartungsgemäß schlechter aus: Weit mehr Praktikanten als Trainees werden nach Praktikumsende nicht übernommen; $31 \%$ der Betriebe haben seit Anfang 2005 überhaupt keinen ihrer Praktikanten anschließend eingestellt. Befürchtet werden kann auch, nach vielen vorangegangenen öffentlichen Klagen, dass 38 \% der Betriebe mit Praktikanten-Einsatz ihren Praktikanten keinerlei Entgelt zahlen und die restlichen - anders als bei Trainees - meist ein qualifikationsunabhängiges Fixum. Gleichzeitig aber bezeichnen die befragten Betriebsräte in $40 \%$ der Betriebe mit Praktikanteneinsatz die jeweilige Praktikantentätigkeit als , eher normale Beschäftigung". Auch die sonstigen Berufseinsteiger erfahren in mehreren Punkten von Betriebsseite nicht die angemessene Wertschätzung. Nur $51 \%$ der Betriebe mit Berufseinsteigern haben für diese einen festen Betreuer, noch weniger (42\%) bieten einen konkreten Betreuungsplan. Und knapp die Hälfte der Betriebe (48 \%) schließt mit Berufseinsteigern ,häufiger befristete Arbeitsverträge als bei Normalbeschäftigten", dehnt also die faktische Probezeit über die in aller Regel ohnehin formal vereinbarte Probezeit aus bzw. bedient das schon erwähnte betriebsseitige Flexibilisierungsinteresse.

Schließlich wird die gesamte Belegschaft häufig stark belastet. In $68 \%$ der befragten Betriebe mit Betriebsrat gibt es bezahlte Mehrarbeit bzw. Überstunden über die tarifliche oder betriebsübliche Arbeitszeit hinaus, im Durchschnitt 3,7 Stunden pro Beschäftigten und Arbeitswoche. In $24 \%$ der Betriebe leisten die Beschäftigten unbezahlte Mehrarbeit, die weder durch Geld noch Zeitguthaben abgegolten wird, durchschnittlich 3,2 Stunden pro Beschäftigten und Arbeitswoche. Selbst der Ausgleich von Mehrarbeit auf Zeitguthaben ist ein Belastungsindikator, wenn in rund $20 \%$ der befragten Betriebe Zeitguthaben nach Erreichen von Grenzwerten bzw. Aus- gleichzeiträumen ersatzlos verfallen - und zwar dort im Durchschnitt pro Beschäftigtem und Beschäftigungsjahr 19,7 Stunden, also eine halbe unbezahlte Arbeitswoche. Aber auch unabhängig davon sind Langzeitkonten bzw. das entsprechende Ansammeln von Zeitguthaben wegen Überstunden nicht ohne materielle Risiken: Trotz gesetzlicher Verpflichtung sind $25 \%$ der befragten Betriebe ohne Insolvenzsicherung von Langzeitkonten; und bei weiteren $13 \%$ sind sich die Betriebsräte über die Existenz einer entsprechenden Absicherung nicht sicher. Angesichts dieser Defizite sind die aktuellen Bemühungen des Bundesarbeitsministeriums zum besseren Insolvenzschutz von Arbeitszeitkonten im Rahmen eines Gesetzentwurfs aus gewerkschaftlicher Sicht nicht ausreichend (DGB 2008) - selbst wenn sich die betriebliche Praxis gegenüber den Befunden der letzten WSI-Befragung (Schietinger 2005) verbessert hat.

Die Belastung durch Mehrarbeit ist umso problematischer, weil die reguläre betriebliche Arbeitszeit in den letzten Jahren ausgedehnt wurde: Nach Auskunft der befragten Betriebsräte gilt dies seit Anfang 2005 für gut $25 \%$ der Betriebe (Betriebsrätebefragung 2005: 15,5\%). Die Arbeitszeitverlängerung blieb bei der Hälfte der Betriebe ohne zusätzliche Bezahlung der Beschäftigten. Parallel sank der Anteil der Betriebe, in denen die reguläre Arbeitszeit verkürzt wurde, von fast $12 \%$ auf knapp $8 \%$. Damit zeigt sich erneut, dass der konjunkturelle Aufschwung weder als Mehr an Beschäftigung noch an Einkommen oder besseren Arbeitsbedingungen befriedigend ankommt.

Gesundheitliche Belastungen und personalpolitische Defizite im Betrieb lassen die befragten Betriebsräte sehr skeptisch beurteilen, ob ihre Beschäftigten ein langes und kürzlich noch verlängertes Arbeitsleben durchhalten können. Dass alle Beschäftigten auf ihren Arbeitsplätzen die neue Regelaltersgrenze von 67 Jahren erreichen können, glauben nur $34 \%$ der Betriebsräte - im privaten Kredit- und Versicherungswesen mit $69 \%$ erheblich mehr, im Baugewerbe mit $16 \%$ deutlich weniger. $14 \%$ der Betriebsräte halten Arbeiten bis zum 67. Lebensjahr in ihrem Betrieb sogar generell für unmöglich. 


\section{BETRIEBSRAT, ARBEITGEBER, GEWERKSCHAFT}

Die Beziehung zwischen Arbeitgeber und Betriebsrat scheint im Zeitvergleich wenig verändert - trotz Aufschwung. Dies belegen z. B. die folgenden Indikatoren: Die gesetzlich vorgeschriebene Freistellung von Betriebsräten wird in $44 \%$ der befragten Betriebe genau eingehalten; in $21 \%$ wird Freistellung über den gesetzlichen Anspruch hinaus gewährt; in $34 \%$ der Betriebe wird der gesetzliche Anspruch nicht ausgeschöpft. $30 \%$ der befragten Betriebsräte erhalten ihnen zustehende Informationen vom Arbeitgeber meist unaufgefordert, $36 \%$ bei der ersten Anfrage und weitere $34 \%$ erst nach mehrfachen Bitten. Die Betriebsräte melden etwas häufiger Versuche der Arbeitgeber, ihre Mitwirkungsrechte zu „behindern“ (39\% - nie; $46 \%$ - manchmal; $14 \%$ - häufig).

Die gewerkschaftliche Unterstützung für Betriebsräte, die zugleich über die Qualität des Verhältnisses Betriebsrat-Gewerkschaft Aufschluss geben kann, wurde in dieser Welle erstmals erfragt. $84 \%$ der befragten Betriebsräte erhalten telefonische oder schriftliche Auskünfte von Gewerkschaften. Dabei wächst die Auskunftshäufigkeit mit der Betriebsgröße ( $82 \%$ in kleinen Betrieben bis 49 Beschäftigten, $95 \%$ in Großbetrieben ab 2.000 Beschäftigten). "Großbetriebs"-Räte mögen fordernder und initiativer sein, aber realistischerweise muss auch ein Großbetriebs-Bias bei den Gewerkschaften angenommen werden.
Weitere gewerkschaftliche Hilfen für Betriebsräte sind: Schulungen und Seminare (nutzen $75 \%$ von ihnen), Beratung bei betrieblichen Konflikten (75\%), Besprechungen mit dem Betriebsrat (69\%), Besuch von Gewerkschaftsvertretern in Betriebsversammlungen (65\%), Unterstützung bei den Betriebsratswahlen (61\%) und Vermittlung von Experten (57\%). In vielen Fällen - auch bei der Unterstützung von Betriebsratswahlen - ist ein Großbetriebs-Bias zu beobachten. Die Kehrseite dieser Befunde ist, dass offensichtlich auch breite Bereiche von Betrieben mit Betriebsrat existieren, die wenig bis gar keine gewerkschaftliche Unterstützung erhalten und vielleicht auch nicht fordern. Die gewerkschaftliche Betreuung wird im Übrigen von den Betriebsräten heute nahezu genauso wie 2005 bewertet: $16 \%$ bezeichnen sie als „sehr gut", $34 \%$ als „gut", $21 \%$ als „befriedigend“, $10 \%$ als „ausreichend“ und $13 \%$ als „mangelhaft“.

\section{1 \\ Fazit und Ausblick}

Wie die früheren Befragungsergebnisse erlauben es auch die aktuell vorliegenden erneut, die Arbeit von Interessenvertretungen als außerordentlich vielfältig, komplex, anpassungsfähig und anspruchsvoll zu bezeichnen. Angesichts der Gesamtzahl der vom IAB ermittelten Betriebsratsgremien von rund 120.000 und der dort versam- melten umso größeren Zahl von Betriebsratsmitgliedern sind sie jedenfalls ein elementares Struktur- und Stabilisierungselement von Wirtschaft und Gesellschaft in Deutschland. Es kann zugleich bezweifelt werden, dass der Wert dieser Arbeit in der Öffentlichkeit angemessen geschätzt wird. Wenige Skandale um korrupte Betriebsräte erschweren zusätzlich ein besseres öffentliches Urteil. Es wäre dafür unter Umständen schon viel gewonnen, wenn es in Deutschland, wie z. B. in Holland, ein Betriebsräteregister mit obligatorischen Meldungen über Neukonstituierung und Mitgliederveränderung geben würde, das Öffentlichkeit und Politik zumindest die quantitative Dimension dieser Arbeit genauer und öfter vor Augen führt.

Auch wenn die Befragungen des WSI schon jetzt erheblich zur Transparenz betrieblicher Mitbestimmung beitragen, ist sie noch lange nicht voll gegeben. Auch deshalb ist die nächste Sonderbefragung schon in Vorbereitung. Sie wird im Herbst 2008 den betrieblichen Komplexen „Gesundheitsschutz bzw. Belastungsprävention“ sowie „Innovationsprozesse“ gelten. Beide Themen werden teilweise im Forschungsverbund mit dem BMBF-geförderten Projekt „Partizipatives Gesundheitsmanagement" (www.pargema.de) des ISF München, der Universität Jena und anderen sowie dem HBS-geförderten Projekt „Innovationstreiber Mitbestimmung" beim Institut für angewandte Innovationsforschung an der Ruhr-Universität Bochum betrieben.

\section{LITERATUR}

DGB (2008): DGB fordert Nachbesserungen - Insolvenzschutz von Arbeitszeitkonten, in: einblick 8, S. 3

Ellguth, P./Kohaut, S. (2007): Tarifbindung und betriebliche Interessenvertretung - aktuelle Ergebnisse aus dem IAB-Betriebspanel 2006, in: WSI-Mitteilungen 9, S. 511-514

Schäfer, C. (2005): Die WSI-Befragung von Betriebs- und Personalräten 2004/05 - ein Überblick, in: WSI-Mitteilungen 6, S. 291-300
Schietinger, M. (2005): Insolvenzsicherung von Arbeitszeitguthaben: Wer trägt das Risiko der Arbeitszeitflexibilisierung?, in: WSI-Mitteilungen 6, S. 339-345

Wassermann, W./Rudolf, W. (2007): Leiharbeit als Gegenstand betrieblicher Mitbestimmung. Ergebnisse einer Befragung von Betriebsräten in 80 Betrieben des Organisationsbereichs der IG Metall im Frühjahr 2007, in: Arbeitspapier 148 der Hans-Böckler-Stiftung, Düsseldorf 